Research Paper

\title{
Sub-MIC of antibiotics induced biofilm formation of Pseudomonas aeruginosa in the presence of chlorhexidine
}

\author{
Safaa T. Aka, Sayran H. Haji \\ Department of Pharmacognosy, College of Pharmacy, Hawler Medical University, Erbil City, Iraq.
}

Submitted: March 12, 2014; Approved: June 6, 2014.

\begin{abstract}
Public health is facing a new challenge due to the alarming increase in bacterial resistance to most of the conventional antibacterial agents. It has been found that only minor cell damage is caused when exposed to sub-lethal levels of antimicrobial. Biofilms can play an important role in producing resistance, which is developed to reservoirs of pathogens in the hospital and cannot be easily removed. The aim of this study was to test whether the sub-lethal dose of antibiotics can induce biofilm formation of $P$. aeruginosa following incubating in the presence and absence of chlorhexidine. Standard antibiotic-micro broth 96-flat well plates were used for determination of MIC and biofilm assay. The adherence degree of biofilm was determined by estimation of $\mathrm{OD}_{630 \mathrm{~nm}}$ values using ELISA reader. The mean 22 isolates of $P$. aeruginosa growing in culture with presence and absence of chlorhexidine, could exhibited the significant $(\mathrm{p}<0.001)$ proportion of adherence followed incubation in sub minimal inhibitory concentrations (Sub-MIC) of cefotaxim, amoxicillin, and azithromycin in comparison with control (antibiotic-free broth), while the sub-MIC of ciprofloxacin revealed significant inhibition of biofilm. Conclusion: Incubating the isolates of $P$. aeruginosa to sub-MIC of antibiotics exhibited induction of biofilm in the presence of chlorhexidine.
\end{abstract}

Key words: antibiotic, biofilm, sub-MIC, chlorhexidine, $P$. aeruginosa.

\section{Introduction}

Antibiotics have been used to treat patients with infectious diseases. They target essential bacterial structures and cellular pathways, such as the cell wall, DNA, RNA, protein synthesis mechanism, and bacterial metabolism. However, long-term use of antibiotics results in the adaptation and development of resistance leading to treatment failure, prolonged hospitalization, increased costs of care, and increased mortality (Masadeh et al., 2012).

Public health is facing a new challenge due to the alarming increase in bacterial resistance to most of the conventional antibacterial agents as well as the emerging link between the resistance strategies employed by bacteria toward antibiotics and biocides (Braoudaki and Hilton, 2004). An antibacterial effect can be defined as an interaction between an active substance and specific targets in the microbial cell (Denyer and Stewart, 1998). Bacterial exposure to a harmful environment will use all their power to survive. External stress has different effects on organisms, leading to natural responses like inhibition or inactivation of the cells. It has been found that only minor cell damage is caused when exposed to sub-lethal levels of antimicrobial. The consequences of that may include changes in their phenotype and induction of gene expression, giving rise to a more resistant population (Russell, 2003);(Araújo et al., 2011). Therefore, resistance mechanisms are the means that living organisms have to respond to continuously changing environment in order to survive (Russell, 2003).

In general, there are three documented types of resistance: inherent resistance, also termed natural or intrinsic; acquired resistance, due to the occurrence of a mutation, and usually mediated by plasmids; and finally, resistance by adaptation which occurs when a community of bacteria acquires resistance to an antimicrobial, it may also acquire resistance to other antimicrobials of the same type (cross resistance) (Chapman, 2003). On the other hand, biofilms can play an important role in producing resistance, which is 
developed to reservoirs of pathogens in the hospital and cannot be easily removed. They are responsible for approximately $65 \%$ of nosocomial infections, thus, developing effective procedures to combat biofilms in the hospital environment to control hospital-acquired infections is of critical importance (Oz et al., 2012).

A biofilm comprises a functional consortium of cells enveloped within a matrix of extracellular polysaccharides. Surface association allows the organisms to persist in a favorable environment, while the biofilm structure protects cells from dehydration and other environmental pressures (Smith and Hunter, 2008). Biofilms can form on almost any biological or a biotic surface and generally have susceptibilities towards antibiotics and biocides that are $100-1000$-fold less than equivalent populations of planktonic bacteria (Gilbert et al., 2002).

Antiseptics and disinfectants are used extensively in hospitals and other health care settings for a variety of topical and hard-surface applications. In particular, they are an essential part of infection control practices and aid in the prevention of nosocomial infections (McDonnell and Russell, 1999). The mode of action of Quaternary ammonium compounds (QACs) is attributed to their positive charge, which forms an electrostatic bond with negatively charged sites on microbial cell walls (McDonnell and Russell, 1999). QACs cause also cell death by protein denaturation, disruption of cell-wall permeability and reduction of the normal intake of life-sustaining nutrients to the cell (Simões et al., 2005).

Biocides are widely used in health-related activities as a convenient means of disinfection and protection against bacterial contamination yet; they carry a risk of resistance to them-selves as well as cross- resistance to antibiotics. The mechanisms by which bacteria resist killing by antibiotics and biocides are still poorly defined, although repeated exposure to sub lethal concentrations of antibacterial agents undoubtedly contributes to their development (Braoudaki and Hilton, 2004). Many studies have been performed in order to access microbial resistance to biocides (Zhang and Mah, 2008). Therefore, the aim of this study was to test whether the sub-MIC of antibiotics can induce biofilm formation of $P$. aeruginosa following incubating in the presence and absence of chlorhexidine

\section{Materials and Methods}

\section{Bacterial isolates}

Twenty two clinical isolates of Pseudomonas aeruginosa were collected from the lab of Rizgary Teaching Hospital in Erbil, Iraq. The origin of isolates was from specimens of ear infections.

\section{Antimicrobial agent and biocicides}

The following antimicrobial agents were selected due to the commonly used in the hospital, which recommended by physicians for treatment of ear infections. Therefore the study evaluated the bacterial resistance against these antibiotics: cefotaxime (CTX) at a concentration of $1000 \mathrm{mg} / \mathrm{mL}$, amoxicillin (AMX) at $500 \mathrm{mg} / \mathrm{mL}$, azithromycin (AZM) at $250 \mathrm{mg} / \mathrm{mL}$, and ciprofloxacin (CIP) at $500 \mathrm{mg} / \mathrm{mL}$. All antibiotics were used as raw material, and purchased from Mepha/Switzerland. Biocide \%4 (w/v) chlorhexidine (CHX) purchased from Al-Rhma pharmaceutical Co. as a laboratory standard solution. All solutions were filter sterilized using a $0.2 \mu \mathrm{m}$ cellulose syringe filter (jet-biofilm, China).

\section{Inoculums preparation}

The MIC and Sub-MIC for the tested CHX were determined, using two-fold tube dilution method. The two stock cultures of bacterial isolates were prepared to the test. The first culture was incubated in the presence of sub lethal doses of CHX, which marked as isolate in the presence of CHX (CHX-culture), while the second culture was incubated in the broth that absence CHX broth and marked as control or (CHX-free culture). These stock cultures were incubated for $72 \mathrm{~h}$ at $37^{\circ} \mathrm{C}$, then bacterial suspension was adjusted to $1 \times 10^{8} \mathrm{cfu} / \mathrm{mL}$ using McFarland 0.5 (biomerieux).

\section{Determination of sub-minimum inhibitory concentrations (sub-MICs) of antibiotics}

Standard antibiotic-micro broth 96-flat well plates were used. Two-fold serial dilutions, ranged from 0.5 to $512 \mu \mathrm{g} / \mathrm{mL}$ for the four types of antibiotics were prepared. The concentrations of antibiotics dispensed on the wells ( $200 \mu \mathrm{L}$ per well). Two sets per each antibiotic in separated microtiter plate were arranged. Control plates were prepared as two sets of free antibiotic-microtiter plate that were all wells dispensed with $200 \mu \mathrm{L}$ of nutrient broth without antibiotics.

\section{Bacterial inoculation and biofilm determination}

Inoculation of plates was achieved by dispensing stock cultures to microtitier wells in subsequent procedure. All wells in the first antibiotic-broth plate were dispensed with $50 \mu \mathrm{L}$ of CHX-culture, while the wells of the second antibiotic-broth plate were inoculated with $50 \mu \mathrm{L}$ of CHXfree culture. The same procedure was repeated to the two antibiotic-free broth plates as control. The total four plates were incubated for $72 \mathrm{~h}$ at $37^{\circ} \mathrm{C}$. In vitro quantitative assessment of biofilm formation was determined; more than one sub-MIC was evaluated as low concentrations dose stimulation of biofilm (Kaplan, 2011). Following incubation, the plates were washed three times by PBS pH 7.2, then exposed to air-dry. A $200 \mu \mathrm{L}$ of $0.1 \%$ crystal violet was added to each well and the plates were incubated at room temperature for $30 \mathrm{~min}$. The plates were washed off using distilled water and kept for air dry. The bound bacte- 
ria were quantified by addition of ethanol $70 \%$ and measurement of the dissolved crystal violet at optical density (OD) of $630 \mathrm{~nm}$ using 96-flat wells microtiter plate of the ELISA reader ELX800 (Biotek / USA). Tests were performed in triplicate for each antibiotic (Braoudaki and Hilton, 2004). The biofilm value was estimated using the following formula:

$$
\text { Biofilm value }=\left(\text { Test } \mathrm{OD}_{630 \mathrm{~nm}}-\text { Control } \mathrm{OD}_{630 \mathrm{~nm}}\right)
$$

\section{Statistical analysis of data}

The mean \pm SD of biofilm to the four different conditions were measured and the paired sample t-test was used to compare these means.

\section{Results}

The adherence degree of biofilm was determined by estimation of OD values for 22 isolates of $P$. aeruginosa grown in various environmental conditions, which included planktonic culture (free living), antibiotic-broth media, and antibiotic-broth in the presence CHX media.

The 22 isolates exhibited greatest growth on all surfaces but the degree of adherence varied among the isolates. Biofilms formation after $72 \mathrm{~h}$ by isolates of CHX-culture were stronger than isolates of CHX-free culture, but the adherence was not statistically significant $(p=0.065)$ as shown in Table 1.

The next stage of this study evaluated the adherence degree to the 22 isolates of $P$. aeruginosa in the absence of $\mathrm{CHX}$ as previously marked CHX-free culture. Following $72 \mathrm{~h}$ exposures to sub-MIC of antibiotics, the biofilm grown on the plates exhibited the highest proportion of adherence for CTX, AMX, and AZM, which displayed statistically significant difference $(p<0.001)$, while CIP showed significant inhibition $(\mathrm{p}<0.001)$ of biofilm compared with those CHX-free culture seeded in antibiotic-free broth plates as shown in Table 2.

Table 3 estimated the adherence degree of CHXcultures of $P$. aeruginosa exposed to sub-MIC of antibiotics broth and antibiotic-free broth. The treated isolates exhibited a significantly induction $(p<0.001)$ in biofilm formation for CTX, AMX and AZM, but not for CIP, in comparison with isolates of antibiotic-free broth.

The last field of present study demonstrated a comparison of induced biofilm by sub-MIC of antibiotic-broth in the presence and absence of CHX. The isolates could ex-

Table 1 - Paired sample statistics of $P$. aeruginosa biofim in control with presence of chlorhexidine at sub-MIC concentrations.

\begin{tabular}{lccc}
\hline Control of P.aeruginosa $(\mathrm{n}=22)$ & $\begin{array}{c}(\text { Mean } \pm \mathrm{SD}) \\
\text { Biofilm }\left(\mathrm{O}^{\mathrm{D}} \mathrm{D}_{60 \mathrm{~nm}}\right)\end{array}$ & p-value \\
\hline Pair 1 & CHX-free culture & $0.0719 \pm 0.008$ & 0.065 \\
& CHX-culture & $0.0767 \pm 0.01$ & \\
\hline
\end{tabular}

hibit biofilm in all surfaces, but the degree of adherence showed various OD- values as shows in table 4 . The subMIC of CTX in the presence of CHX marked as (CTX:CHX) displayed a significant difference of ODvalues $(\mathrm{p}<0.001)$ in comparison with (CTX:CHX-free). Even though, the biofilm degree in isolates from AMX:CHX and AZM:CHX showed less degree of adherence, but they exhibited a significant difference compared with their pairs AMX:CHX-free and AZM:CHX-free $(p=0.03$ and $p=0.037$, respectively). The isolates in CIP:CHX revealed no statistically increasing biofilm degree $(\mathrm{p}=0.616)$ compared with CIP:CHX-free.

\section{Discussion}

The study aimed to evaluate a bacterial biofim of 22 $P$. aerugiousa isolates, which induce by sub-MIC of antibiotics in the presence / absence of CHX at sub lethal concentrations. The aim of this work was to estimate the factors or environmental conditions that may enhance bacterial biofilm. This is because bacteria-forming biofilms are resistant to killing by antibiotics 10.000 times greater than concentration needed to kill "Planktonic" cell (Mah and O'Toole, 2001).

Many bacterial isolates in hospital environment can colonize all surfaces as biofilms, creating a reservoir for infection for hospitalized individuals (Vincent, 2003). Studies suggested that bacterial biofilm are responsible for $65 \%$ of hospital acquired infection (Potera, 1999). In this study the efficacy of commonly used antibiotics at the sub-MIC concentration on biofilm formation were determined using clinical isolates of $P$. aeruginosa grown in liquid culture in the presence / absence of CHX. Both bacterial isolates (CHX-culture) and (CHX-free culture) incubated for $72 \mathrm{~h}$, could form biofilm following cultivation in antibiotic-free broth. In fact, the OD values showed greater biofilm, which enhanced by CHX-culture compared with CHX-free culture, although the difference was not statistically significant. These cells may started to show resistance mechanism to survive the attack due to changes the phenotypic level, i.e. the ability to form biofilm, which is an adaptive form of resistance (Poole, 2004).

On the other hand, the bacterial isolates showed a significantly increased adherence degree to surfaces following exposure to sub-MIC of antibiotics. These results come consistent and inconsistent with previous works. Several studies have shown that sub-MIC of some antibiotics that are unable to kill bacteria, can inhibit biofilm formation, azithromycin was a good example, which efficiently inhibits $P$. aeruginosa biofilm formation at low concentrations (Wagner et al., 2005). In contrast, numerous studies have shown that some antibiotics at their low concentrations could significantly induce biofilm formation in variety of bacterial species (Kaplan, 2011). This process may have clinical relevance because bacteria are exposed to low concentrations of antibiotics at the beginning and end of the 
Table 2 - Paired sample statistics of biofilm at sub-MIC of antibiotic broth and antibiotic-free broth against CHX-free culture of P.aeruginosa.

\begin{tabular}{lcc}
\hline $\begin{array}{l}\text { Sub-MIC antibiotics and antibiotics-free broth against CHX-free culture } \\
\text { of } P \text {. aeruginosa }(\mathrm{n}=22)\end{array}$ & (Mean \pm SD) Biofilm $\left(\mathrm{O} . \mathrm{D}_{630 \mathrm{~nm}}\right)$ \\
\hline Pair 1 & CTX-free broth & $0.0719 \pm 0.008$ \\
& CTX-broth & $0.0812 \pm 0.01$ \\
Pair 2 & AMX-free broth & $0.0719 \pm 0.008$ \\
& AMX-broth & $0.0824 \pm 0.01$ \\
Pair 3 & CIP-free broth & $0.0719 \pm 0.008$ \\
& CIP-broth & $0.0589 \pm 0.007$ \\
Pair 4 & AZM-free broth & $0.001 *$ \\
& AZM-broth & $0.0719 \pm 0.008$ \\
\hline
\end{tabular}

* Significant inhibition of biofilm.

Table 3 - Paired sample statistics of biofilm at sub-MIC of antibiotic broth and antibiotic-free broth against CHX-culture of P.aeruginosa.

\begin{tabular}{lcc}
\hline $\begin{array}{l}\text { Sub-MIC antibiotics and antibiotics-free broth against CHX-culture of } \\
P . \text { aeruginosa }(\mathrm{n}=22)\end{array}$ & (Mean $\pm \mathrm{SD})$ Biofilm $\left(\mathrm{O} . \mathrm{D}_{630 \mathrm{~nm}}\right)$ \\
\hline Pair 1 & CTX-free broth & $0.0767 \pm 0.01$ \\
& CTX-broth & $0.1002 \pm 0.017$ \\
Pair 2 & AMX-free broth & $0.0767 \pm 0.01$ \\
& AMX-broth & $0.0898 \pm 0.017$ \\
Pair 3 & CIP-free broth & $0.0767 \pm 0.01$ \\
& CIP-broth & $0.06 \pm 0.009$ \\
Pair 4 & AZM-free broth & $0.0767 \pm 0.01$ \\
& AZM-broth & $0.0938 \pm 0.014$ \\
\hline
\end{tabular}

*Significant inhibition of biofilm.

Table 4 - Paired sample statistics of biofilm at sub-MIC of antibiotic broth against CHX-culture and CHX-free culture of $P$. aeruginosa.

\begin{tabular}{lcc}
\hline $\begin{array}{l}\text { Sub-MIC antibiotics against CHX-culture and CHX-free culture of } \\
\text { P.aeruginosa }(\mathrm{n}=22)\end{array}$ & (Mean \pm SD) Biofilm $\left(\mathrm{O}_{\text {.D }}\right.$ 630 $\left.\mathrm{nm}\right)$ \\
\hline Pair 1 & CTX:CHX-free & $0.0812 \pm 0.012$ \\
& CTX:CHX & $0.1002 \pm 0.017$ \\
Pair 2 & AMX:CHX-free & $0.0824 \pm 0.01$ \\
& AMX:CHX & $0.0898 \pm 0.017$ \\
Pair 3 & CIP:CHX-free & $0.0589 \pm 0.007$ \\
& CIP:CHX & $0.06 \pm 0.009$ \\
Pair 4 & AZM:CHX-free & 0.016 \\
& AZM:CHX & $0.0875 \pm 0.016$ \\
\hline
\end{tabular}

dosing treatment or in case of continuously low doses therapy.

The study selected wide range of sub-MIC to detect biofilm induction, and not just concentrations below MIC, since studies failed to demonstrate biofilm induction by bacterial isolates in response to (1/2 MIC), but could exhibit maximal biofilm induction at 1/4 MIC (Kaplan et al., 2011). Many studies investigating the mechanisms of anti- biotic-induced biofilm formation have carried out on pathogens such as P. aeruginosa, Staphylococci and $E$. coli. It has been found that not all antibiotics could induce biofilm, even if these antibiotics are belong to the same group. For example members of $\beta$-lactams could induce $c p s$ gene, which is required for synthesis of colanic acid and further increased capsular polysaccharide in E. coli, but expression of cps gene was not induced by other 
$\beta$-lactams (Sailer et al., 2003). Moreover, studies on $P$. aeruginosa, demonstrated that different groups with the varied mode of action could reveal different effect on biofilm formation, for instance sub-MIC of aminoglycosides induced $P$. aeruginosa biofilm formation but membraneactive peptide, chloramphenicol and cell wall synthesis inhibitors, had no effect on biofilm formation (Hoffman et $a l ., 2005)$. These findings agreed with our results that revealed inhibitory effect of sub-MIC of CTX, AMX and AZM induced biofilm formation, while CIP reduced or inhibited biofilm.

Several studies have shown the sub-MIC antibiotic could induce $P$. aeruginosa biofilm formation through mechanisms that involve the intracellular second messenger cyclic dimeric guanosine monophosphate (c-diGMP)(Mah and O'Toole, 2001). In fact, increased c-diGMP level generally results in an increase in exopolysaccharide; fimbria production and decrease motility, then increased biofilm formation (Yildiz, 2008). The study showed AMX and CTX as members of $\beta$-lactams induced biofilm formation of $P$. aeruginosa isolates exposed to $1 / 4$ $\mathrm{MIC}$, this effect might be due to gene expression, since studies found that more than one alg gene that involve in alginate metabolism, were induced more than 10-fold (Bagge et al., 2004). Furthermore, biofilm inhibition by $P$. aeruginosa in response to antibiotics is consistent with hypothesis that metabolic stress is the key signal that mediates the response (Boehm et al., 2009). The study also showed antibiotic induced biofilm formation, which also agrees with finding of Linares et al. (Linares et al., 2006), who found that sub-MIC of antibiotic induced $P$. aeruginosa biofilm by approximately 2 -fold, suggesting the antibiotic caused significant increase in the expression up to $7 \%$ of 555 genes in genomic array selected as relevant for development of colonization and further stress response that had been shown to play a role in biofilm formation.

This study demonstrated the sub-MIC of AZM induced biofilm formation. Indeed, several studies disagreed with these results, in view of the fact they suggested subMIC of AZM could alter or inhibit biofilm developed by $P$. aeruginosa. (Nagino and Kobayashi, 1997);(Saiman et al., 2003);(Borriello et al., 2004);(Fux et al., 2005) In contrast to these results, Gillis and Iglewski (Gillis and Iglewski, 2004) could reveal that sub-MIC of AZM appear to be specific to initial stages of biofilm development since after 48 $h$, a resistance phenotype was able to weaken the inhibitory effect of AZM and result in a very strong biofilm. Thus the static model system may not have provided sufficient time for a biofilm variant to develop and be detected. These finding suggest that AZM may exhibit different biofilm inducing activities against $P$. aeruginosa (Kaplan, 2011).

The last line of the study showed that isolates of $P$. aeruginosa following incubation in sub inhibitory concentrations of CHX could induce stronger biofilm in the presence of sub-MIC of antibiotics. In fact, the outer mem- brane of $P$. aeruginosa is responsible for this resistance to $\mathrm{CHX}$ and many other antiseptics. The high $\mathrm{Mg}^{+2}$ content aids in producing strong LPS-LPS link; furthermore, the porins may act as a barrier to prevent their diffusion (McDonnell and Russell, 1999). This physiological adaptation support many bacterial isolates associated with solid surface to generate a biofilm (Costerton et al., 1994). These results could support our findings of antibiotic induced biofilm formation in the presence of sub lethal doses of $\mathrm{CHX}$. Therefore, phenotypic change by $\mathrm{CHX}$ and induction of gene expression due to antibiotics action might enhance bacterial resistant and further stronger biofilm formation. Thus, resistance mechanisms are the means that living organisms have to respond to continuously changing environment in order to survive (Russell, 2003, Araújo et $a l ., 2011)$. The study concluded that incubating the isolates of $P$. aeruginosa in sub-MIC of antibiotics exhibited induction of biofilm in the presence of chlorhexidine. Therefore, this study will help establish the medical application to guide antibiotic therapy and hospital disinfection that would suppress the biofilm induction.

\section{References}

Araújo P, Lemos M, Mergulhão F et al. (2011) Antimicrobial resistance to disinfectants in biofilms. In: A. Méndez-Vilas (ed) Science Against Microbial Pathogens: Communicating Current Research and Technological Advances. Formatex, Badajoz, pp 826-834.

Bagge N, Schuster M, Hentzer M et al. (2004) Pseudomonas aeruginosa biofilms exposed to imipenem exhibit changes in global gene expression and $\beta$-lactamase and alginate production. Antimicrob Agents Chemotherapy 48:1175-1187.

Boehm A, Steiner S, Zaehringer F et al. (2009) Second messenger signalling governs Escherichia coli biofilm induction upon ribosomal stress. Mol Microbiol 72:1500-1516.

Borriello G, Werner E, Roe F et al. (2004) Oxygen limitation contributes to antibiotic tolerance of Pseudomonas aeruginosa in biofilms. Antimicrob Agents Chemother 48:2659-2664.

Braoudaki M, Hilton AC (2004) Low level of cross-resistance between triclosan and antibiotics in Escherichia coli K-12 and E. coli $\mathrm{O} 55$ compared to E. coli O157. FEMS Microbiol Lett 235:305-309.

Chapman JS (2003) Biocide resistance mechanisms. Int Biodeterior Biodegradation 51:133-138.

Costerton J, Lewandowski Z, DeBeer D et al. (1994) Biofilms, the customized microniche. J Bacteriol 176:2137.

Denyer SP, Stewart G (1998) Mechanisms of action of disinfectants. International Biodeterioration and Biodegradation 41:261-268.

Fux C, Costerton J, Stewart P et al. (2005) Survival strategies of infectious biofilms. Trends Microbiol 13:34-40.

Gilbert P, Allison D, McBain A (2002) Biofilms in vitro and in vivo: do singular mechanisms imply cross-resistance? J Appl Microbiol 92:98S-110S.

Gillis RJ, Iglewski BH (2004) Azithromycin retards Pseudomonas aeruginosa biofilm formation. $\mathrm{J}$ Clin Microbiol 42:5842-5845. 
Hoffman LR, D'Argenio DA, MacCoss MJ et al. (2005) Aminoglycoside antibiotics induce bacterial biofilm formation. Nature 436:1171-1175.

Kaplan JB (2011) Antibiotic-induced biofilm formation. Int J Artif Organs 34:737-751.

Kaplan JB, Jabbouri S, Sadovskaya I (2011) Extracellular DNAdependent biofilm formation by Staphylococcus epidermidis RP62A in response to subminimal inhibitory concentrations of antibiotics. Res Microbiol 162:535-541.

Linares J, Gustafsson I, Baquero F et al. (2006) Antibiotics as intermicrobial signaling agents instead of weapons. Proc Natl Acad Sci 103:19484-19489.

Mah T-FC, O'Toole GA (2001) Mechanisms of biofilm resistance to antimicrobial agents. Trends Microbiol 9:34-39.

Masadeh MM, Mhaidat NM, Alzoubi KH et al. (2012) In vitro determination of the antibiotic susceptibility of biofilmforming Pseudomonas aeruginosa and Staphylococcus aureus: possible role of proteolytic activity and membrane lipopolysaccharide. Infect Drug Res 6:27-32.

McDonnell G, Russell AD (1999) Antiseptics and disinfectants: activity, action, and resistance. Clin Microbiol Rev 12:147-179.

Nagino K, Kobayashi H (1997) Influence of macrolides on mucoid alginate biosynthetic enzyme from Pseudomonas aeruginosa. Clin Microbiol Infect 3:432-439.

Oz Y, Dag I, Kiraz N (2012) Efficacy of Disinfectants on Candida Biofilms at Different Concentrations and Contact Times. B M Research Journal 2:40-52.

Poole K (2004) Efflux-mediated multiresistance in Gram-negative bacteria. Clin Microbiol Infect 10:12-26.

Potera C (1999) Forging a link between biofilms and disease. Science 283:1837-1839.
Russell A (2003) Biocide use and antibiotic resistance: the relevance of laboratory findings to clinical and environmental situations. Lancet Infect Dis 3:794-803.

Sailer FC, Meberg BM, Young KD (2003) $\beta$-Lactam induction of colanic acid gene expression in Escherichia coli. FEMS Microbiol Lett 226:245-249.

Saiman L, Marshall BC, Mayer-Hamblett N et al. (2003) Azithromycin in patients with cystic fibrosis chronically infected with Pseudomonas aeruginosa: a randomized controlled trial. JAMA 290:1749-1756.

Simões M, Pereira MO, Vieira MJ (2005) Action of a cationic surfactant on the activity and removal of bacterial biofilms formed under different flow regimes. Water Res 39:478486.

Smith K, Hunter IS (2008) Efficacy of common hospital biocides with biofilms of multi-drug resistant clinical isolates. J Med Microbiol 57:966-973.

Vincent J-L (2003) Nosocomial infections in adult intensive-care units. Lancet 361:2068-2077.

Wagner T, Soong G, Sokol S et al. (2005) Effects of azithromycin on clinical isolates of Pseudomonas aeruginosa from cystic fibrosis patients. CHEST J 128:912-919.

Yildiz FH (2008) Cyclic dimeric GMP signaling and regulation of surface-associated developmental programs. J Bacteriol 190:781-783.

Zhang L, Mah T-F (2008) Involvement of a novel efflux system in biofilm-specific resistance to antibiotics. J Bacteriol 190:4447-4452.

Associate Editor: Roxane Maria Fontes Piazza

All the content of the journal, except where otherwise noted, is licensed under a Creative Commons License CC BY-NC. 